\title{
RECONSTRUCTION OF THE TRANSMISSION COEFFICIENT FOR STEPLIKE FINITE-GAP BACKGROUNDS
}

\author{
IRYNA EGOROVA AND GERALD TESCHL
}

\begin{abstract}
We consider scattering theory for one-dimensional Jacobi operators with respect to steplike quasi-periodic finite-gap backgrounds and show how the transmission coefficient can be reconstructed from minimal scattering data. This generalizes the Poisson-Jensen formula for the classical constant background case.
\end{abstract}

Mathematics subject classification (2000): Primary 30E20, 30F30; Secondary 34L25, 47B36. Keywords and phrases: Jacobi operators, scattering theory, periodic, Abelian integrals.

\section{REFERENCES}

[1] N. I. AkHiEZER, Elements of the Theory of Elliptic Functions, Amer. Math. Soc., Providence, 1990.

[2] A. Boutet De Monvel, I. Egorova, And G. Teschl, Inverse scattering theory for onedimensional Schrödinger operators with steplike finite-gap potentials, J. Analyse Math., 1061 (2008), 271-316.

[3] W. Bulla, F. Gesztesy, H. Holden, And G. Teschl, Algebro-Geometric Quasi-Periodic FiniteGap Solutions of the Toda and Kac-van Moerbeke Hierarchies, Mem. Amer. Math. Soc., 135641 (1998).

[4] V.S. BusLaEV AND V.N. Fomin, An inverse scattering problem for the one-dimensional Schrödinger equation on the entire axis, Vestnik Leningrad. Univ., 171 (1962), 56-64.

[5] P. DeIFT AND X. ZHou, A steepest descent method for oscillatory Riemann-Hilbert problems, Ann. of Math. (2), 137 (1993), 295-368.

[6] I. Egorova, J. Michor, AND G. Teschl, Scattering theory for Jacobi operators with quasiperiodic background, Comm. Math. Phys., 2643 (2006), 811-842.

[7] I. EgOROVA, J. MichOR, AND G. TESCHL, Inverse scattering transform for the Toda hierarchy with quasi-periodic background, Proc. Amer. Math. Soc., 135 (2007), 1817-1827.

[8] I. EgOROva, J. Michor, AND G. Teschl, Scattering theory for Jacobi operators with steplike quasi-periodic background, Inverse Problems, 23 (2007), 905-918.

[9] I. EgOROVA, J. Michor, AND G. TESCHL, Soliton solutions of the Toda hierarchy on quasi-periodic background revisited, Math. Nach. 282 4, (2009) 526-539.

[10] I. Egorova, J. Michor, AND G. TESChL, Scattering theory for Jacobi operators with general steplike quasi-periodic background, Zh. Mat. Fiz. Anal. Geom., 41 (2008), 33-62.

[11] H. Farkas And I. Kra, Riemann Surfaces, $2^{\text {nd }}$ edition, GTM 71, Springer, New York, 1992.

[12] F. Gesztesy, H. Holden, J. Michor, And G. Teschl, Soliton Equations and their AlgebroGeometric Solutions. Volume II: $(1+1)$-Dimensional Discrete Models, Cambridge Studies in Advanced Mathematics, 114, Cambridge University Press, Cambridge, 2008.

[13] S. KAMVISsis, On the long time behavior of the doubly infinite Toda lattice under initial data decaying at infinity, Comm. Math. Phys., 1533 (1993), 479-519.

[14] S. KAMVISSIS AND G. TeSCHL, Stability of periodic soliton equations under short range perturbations, Phys. Lett. A, 3646 (2007), 480-483.

[15] S. KAMVISSIS AND G. Teschl, Stability of the periodic Toda lattice under short range perturbations, arXiv:0705.0346

[16] S. KAmvissis And G. Teschl, Stability of the periodic Toda lattice: Higher order asymptotics, arXiv:0805.3847 
[17] R. Killip AND B. Simon, Sum rules for Jacobi matrices and their applications to spectral theory, Ann. of Math.(2), 158 (2003), 253-321.

[18] H. KRÜGER AND G. TESCHL, Long-time asymptotics for the Toda lattice in the soliton region, Math. Z. (to appear).

[19] H. KRÜGER AND G. TeSChL, Long-time asymptotics of the Toda lattice for decaying initial data revisited, Rev. Math. Phys. 21 1, (2009) 61-109.

[20] H. KRÜGER AND G. TESCHL, Long-time asymptotics for the periodic Toda lattice in the soliton region, arXiv:0807.0244 Math. Z. (to appear), DOI : 10.1007/s00209-008-0391-9

[21] A. LAPTEV, S. NABOKO, AND O. SAFRONOv, On new relations between spectral properties of Jacobi matrices and their coefficients, Comm. Math. Phys., 2411 (2003), 91-110.

[22] J. Michor AND G. TESCHL, Trace formulas for Jacobi operators in connection with scattering theory for quasi-periodic background, in Operator Theory, Analysis and Mathematical Physics, J. Janas, et al. (eds.), 51-57, Oper. Theory Adv. Appl., 174 Birkhäuser, Basel, 2007.

[23] F. Nazarov, F. Peherstorfer, A. Volberg, AND P. Yuditskit, On generalized sum rules for Jacobi matrices, Int. Math. Res. Not., 20053 (2005), 155-186.

[24] B. Simon ANd A. Zlatoš, Sum rules and the Szegö condition for orthogonal polynomials on the real line, Comm. Math. Phys., 2423 (2003), 393-423.

[25] G. TESCHL, Oscillation theory and renormalized oscillation theory for Jacobi operators, J. Diff. Eqs., 129 (1996), 532-558.

[26] G. TESCHL, Inverse scattering transform for the Toda hierarchy, Math. Nach., 202 (1999), 163-171.

[27] G. TeSCHL, Jacobi Operators and Completely Integrable Nonlinear Lattices, Math. Surv. and Mon., 72, Amer. Math. Soc., Providence, R.I., 2000.

[28] G. TESCHL, Algebro-geometric constraints on solitons with respect to quasi-periodic backgrounds, Bull. London Math. Soc., 394 (2007), 677-684.

[29] V. Voichick ANd L. Zalcman, Inner and outer functions on Riemann surfaces, Proc. Amer. Math. Soc., 16 (1965), 1200-1204.

[30] A. Volberg AND P. YUditskit, On the inverse scattering problem for Jacobi Matrices with the Spectrum on an Interval, a finite system of intervals or a Cantor set of positive length, Comm. Math. Phys., 226 (2002), 567-605.

[31] A. Zlatoš, Sum rules for Jacobi matrices and divergent Lieb-Thirring sums, J. Funct. Anal., 2252 (2005), 371-382. 